\title{
IMPROVING THE KNOWLEDGE OF OPERATING THEATRE ASSISTANTS
}

\author{
Pinto MV. ${ }^{1}$, Jayakody $S^{2}$,Thennakoon T.M.U.K ${ }^{2}$,Abesundara A.B ${ }^{3}$,Vithanavasam $D^{1}$ \\ ${ }^{1}$ Department of Anaesthesiology, Faculty of Medicine, University of Peradeniya, \\ ${ }^{2}$ Teaching Hospital, Peradeniya, ${ }^{3}$ Teaching Hospital, Kandy \\ *Corresponding author : vasantipinto@yahoo.com
}

Key Words : Operating Department Assistants, Training ODPs

\section{Background:}

Operating Theatre Assistants (OTAs) deliver assistance in anaesthesia, surgery and recovery of patients. They require an adequate knowledge and competent clinical skills. In Sri Lanka this category of assistants does not receive any special training.

\section{Method:}

Operating theatre assistants in two Teaching hospitals in Sri Lanka were evaluated using a questionnaire, which were designed to assess the knowledge of anaesthetic machine and safety features, circuits and gases used in anaesthetic practice, electrical devices and safety, knowledge of resuscitation and airway management and knowledge on vital signs and monitoring.

\section{Results:}

1. $<80 \%$ of required knowledge was evident in $94 \%$ of people with a $\mathrm{p}$ value of $<0.001$

2. The knowledge on circuits and gases used in anaesthetic practice $<80 \%$ of knowledge was seen in $98 \%$ with a significant difference of $\mathrm{p}<0.001$.

3. The knowledge on electrical devices and safety $<80 \%$ knowledge in $82 \%$ of study sample with $\mathrm{P}=0.001$

4. The knowledge of resuscitation and airway management $>80 \%$ of knowledge in $70 \%$ of the sample which did not show a significant difference.

5. The knowledge on vital signs and monitoring >80\% of knowledge in $45 \%$ of them with a non significant difference of the two variables.

6. The educational background of the sample was checked. This revealed that 99.5\% of them were educated up to the GCS O/L. 97.37\% did not have an adequate knowledge of English to understand books and journals.

\section{Conclusion:}

The knowledge of candidates regarding anaesthetic assistance was significantly less than the standard value. Their educational background was adequate to understand a training program conducted in sinhala language.

Within the current health care training system in Sri Lanka it will take many years to achieve this target. We explored the possibility of conducting a program in sinhala language and with available resources. Two training programmes with lectures and hands on sessions were organized and carried out. The evaluation at the end revealed a successful program.

Operating Department Assistants have several occupational names such as Operating
Department Practitioners (ODP), Orderly, Operating Theatre Technician and Theatre 
Support Worker. They are an important part of the operating team working with Surgeons, Anaesthetists and nurses and deliver assistance in aspects of anaesthesia, surgery and recovery of the patient. Their skilled help ensures smooth completion of surgery. Their role involves, the application of theory to practice in various clinical settings. For that they need to have a broad knowledge as well as competent clinical skills. In Sri Lanka we do not have specially trained personnel of this category. The assistants we have are hospital attendants who have gained experience on the job.

A comparison between ODPs in developed countries and Sri Lankan OTAs can be drawn. During the anaesthetic phase, the ODP of a developed country will assist the patients and provide individualized care prior to surgery and anaesthesia. This aspect was not necessarily expected of the Sri Lankan OTAs. The preparation of equipment including anaesthetic machine, intravenous equipment and devices, airway management devices and assistance during securing the airway, is entertained by both categories. But ODPs of developed country are involved with preparation of drugs which is not expected of our OTAs. In developed countries a major role in the recovery unit is played by the ODPs. Receiving the patient, monitoring and assessment involve, the ODPs. In our setting they only deliver the patient to the recovery unit and support to monitor the patient.

The study was carried out

1) To assess the knowledge of our OTAs regarding the anaesthetic assistance.

2) To assess the educational background of the OTAs.

3) To propose a training program to improve the knowledge of OTAs.

\section{Material and Method:}

\section{Objective 1}

An audit was done in two leading teaching hospitals in Sri Lanka with a bed strength of 851 and 1700 and 8 and 13 main operating theatres respectively.

All the operating theatre assistants in the two Teaching hospitals were evaluated using a questionnaire. These questions had been designed to assess the knowledge on,

1. Anaesthetic machine and its safety features.

2. Circuits and gases used in anaesthetic practice.

3. Electrical devices and safety.

4. Knowledge of resuscitation and airway management

5. Vital signs and monitoring.

\section{Objective 2}

A questionnaire was answered regarding the educational background.

\section{Results:}

1. Anaesthetic machine and its safety features.

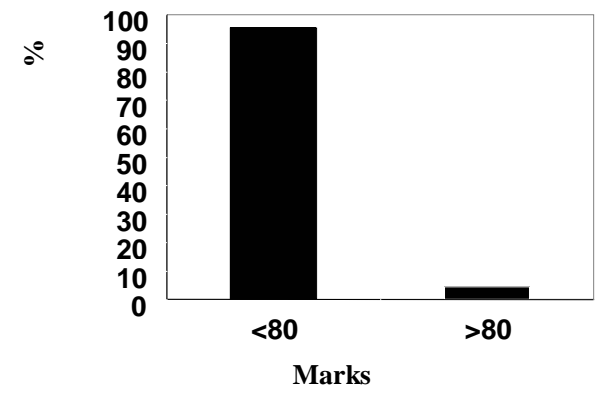

2. Circuits and gases used in anaesthetic practice

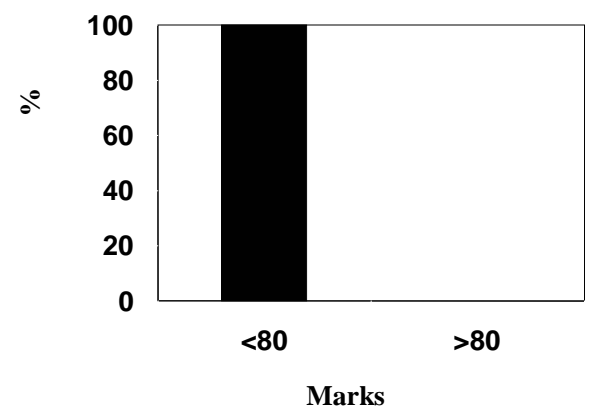

3. Electrical devices and safety

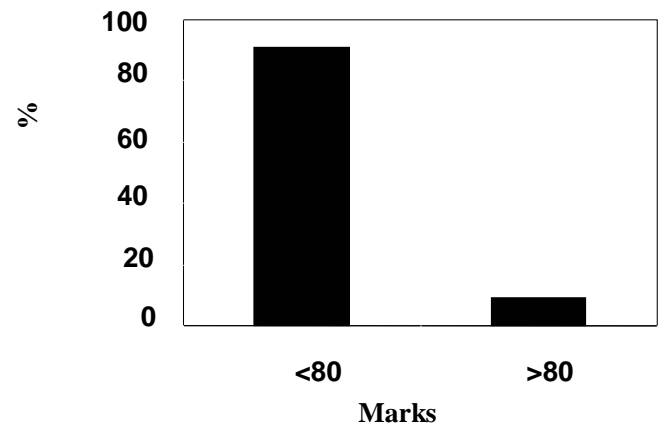


4. Knowledge of resuscitation and airway management

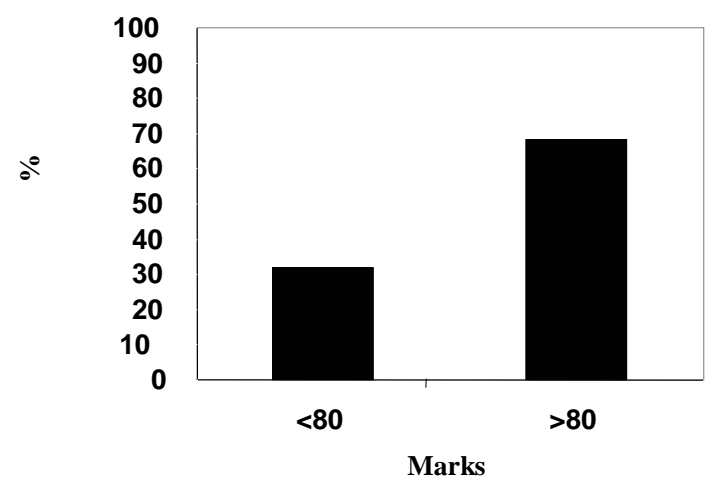

5. Vital signs and monitoring.

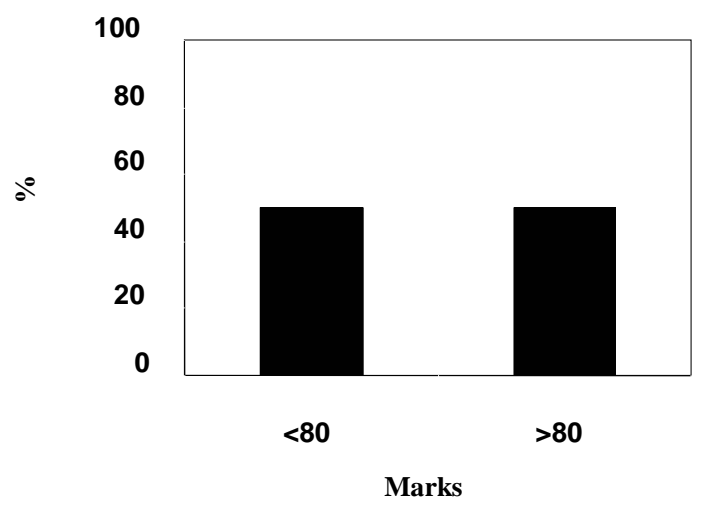

Educational back ground

\begin{tabular}{|l|l|}
\hline \multicolumn{1}{|c|}{$\begin{array}{l}\text { G.C.E A/L } \\
21.05 \%\end{array}$} & $\begin{array}{l}\text { G.C.E O/L } \\
78.95 \%\end{array}$ \\
\hline \multicolumn{2}{|l|}{ English } \\
\hline Credit Pass & $02.63 \%$ \\
\hline Pass & $23.68 \%$ \\
\hline Fail & $73.68 \%$ \\
\hline
\end{tabular}

\section{Discussion:}

Anaesthetists should be vigilant to ensure that the OTAs who help them are properly trained. ${ }^{1} \mathrm{We}$ believe it is mandatory and timely to improve OTAs knowledge in order to provide better patient care in the theatre. They would be capable of helping the anaesthetist in a more competent way than the previously relatively untrained operating theatre labourers.

A full day workshop was organized to train them in the knowledge of anaesthetic assistance. The programme was conducted in sinhala language and comprised of 5 lectures and 4 work stations regarding,

1. Anaesthetic machine and circuits

2. Airway management

3. Resuscitation

4. Monitoring

5. Electrical instruments and safety.

It was conducted by consultant anesthetists, registrars and senior house officers in anaesthesia. An evaluation showed that the programme resulted in a very rewardable outcome and overal encouraging comments.

Similar programmes and training schemes with an examination and evaluation can be conducted at regional levels.

The same audit was extended to check the educational background of the sample by checking the highest educational qualification and the knowledge of the English language. This revealed that in $21.5 \%$ of them GCE/AL was the highest qualification. The GCE $(\mathrm{O} / \mathrm{L})$ was the highest in $78.95 \%$. English as a credit pass was achieved by only $2.63 \%$. $23.68 \%$ got a pass and $73.68 \%$ of them have failed English. The knowledge was significantly less than the required level and also the educational background was well adequate to understand a training programme which needs to be conducted in the sinhala language. Reading material was not available in the Sinhala language. These conclusions revealed the fact that it was mandatory and timely to improve the knowledge of the operating theatre assistance.

The educational standard of the developed country ODP is very high. Their admission requirements for the training program needs a minimum of $5 \mathrm{GCE} / \mathrm{OLs}$ at grades A-C. Some institutions in UK require advanced level qualifications. Their training consist of theory and practicals in the operating theatres. The program generally takes 2 years and lead to a diploma in operating theatre practice.

Within the current health care training system in Sri Lanka we will take very many years to achieve this target. 
We explored the possibility of conducting a program in the native language (Sinhala) and with available resources, making use of the consultant anaesthetists, registrars and senior house Officers in anaesthesia to teach the necessary knowledge at a "hands on" workshop. Two training programmes with lectures and "hands on" sessions were organized and carried out. The evaluation at the end revealed the success of the programme. Reassessment done after 3 months showed the retaining power of the OTAs to be acceptable. We also recommend having similar training programmes every 6 months.

This program was introduced by the College of Anaesthesiologists of Sri Lanka. The detailed training program and the teaching materials were kept available at the college to be used in the future. This programme can be carried out at hospital level, district level or national level to train the OTAs. This would enable the OTAs to be better equipped with knowledge and training to help the Anaesthetists. There will be some possibility of having a salary increment or other benefit for this particular group of people and that will definitely attract more competent attendants to select operating theatre work and subsequent training will definitely improve the outcome.

\section{Conclusion:}

1. The knowledge of the candidates regarding anaesthetic assistance was significantly less than the required value.

2. Their educational background was adequate to understand a training program but it should be conducted in the Sinhala language.

\section{Acknowledgement:}

We wish to thank the College of Anaesthesiologists for the support extended to carry out the training programme and The consultant staff, registras and senior house officers of anaesthesia at Teaching Hospital Peradeniya and Kandy.

\section{References:}

1. Anaesthesia: Editorial notice - 1978; 33; 427-429

$* * * * * * * * * * * * * * * * * * * * * * * * * * * * * * * * * * * * * * * * * * * * * * * * * * * * * * * * * * * * * * * * * * * * * *$

\title{
THE COLLEGE OF ANAESTHESIOLOGISTS OF SRI LANKA
}

\section{CME LECTURE SERIES - 2009}

\author{
Every third Saturday of the month
}

Time: $10.00 \mathrm{am}-12.00$ noon

Venue: Dept of Surgery Lecture Hall, NHSL 